\title{
Mesonephric-like carcinoma of the uterine corpus: A case
}

\section{report and literature review}

\author{
Hyang Sook Jeong ${ }^{1}$, Yuki Gen ${ }^{2}$, Hui Ryun $\mathrm{Joo}^{2}$, Ji Geun Yoo ${ }^{3}$, Seung Geun Yeo ${ }^{4}$, Dong Choon Park ${ }^{2}$ \\ ${ }^{1}$ Department of Hospital Pathology, St. Vincent's Hospital, The Catholic University of Korea, Seoul, South Korea \\ ${ }^{2}$ Department of Obstetrics and Gynecology, St. Vincent's Hospital, The Catholic University of Korea, Seoul, South Korea \\ ${ }^{3}$ Department of Obstetrics and Gynecology, Daejeon St, Mary's Hospital, The Catholic University of Korea, Seoul, South Korea \\ ${ }^{4}$ Medical Science Research Institute, Kyung Hee University Medical Center, Kyung Hee University, Seoul, South Korea
}

\begin{abstract}
Summary
This report describes a woman with uterine mesonephric-like carcinoma and reviews the literature on this rare condition. A 60 -yearold woman was referred for abdominal discomfort. Pelvic magnetic resonance imaging showed a tumor, about $7.0 \mathrm{~cm}$ in diameter in her left ovary. Surgical exploration, however, showed that both ovaries were grossly free of tumor. Rather, a protruding cancerous mass was found on the left side of her uterus. The tumor was composed of cells with various growth patterns and was diffusely positive for PAX8 and GATA3 on immunohistochemistry. The apical luminal border of the glandular structures was positive for CD10, and the glandular structures were focally positive for EMA. The final diagnosis was mesonephric-like carcinoma, stage pT3N1M. Conclusion: Diagnosing mesonephric-like carcinoma is pathologically challenging because these tumors are rare and present with a wide spectrum of morphologic patterns. Positive staining for PAX8 and GATA3 and a luminal staining pattern of CD10 can support the diagnosis.
\end{abstract}

Key words: Mesonephric-like carcinoma; Uterine corpus; Immunohistochemistry.

\section{Introduction}

Malignant mesonephric tumors are rare neoplasms derived from remnants of the mesonephric (Wolffian) ducts in the female genital tract [1]. During embryogenesis, these ducts develop parallel to the müllerian ducts, later becoming the vas deferens and epididymis in males. In females, the mesonephric ducts gradually regress in the absence of testosterone. Mesonephric remnants, which persist in up to $22 \%$ of female adults, are predominantly found in the mesoovaries, broad ligaments, and lateral walls of the cervix [24]. These remnants, however, are uncommon in the uterine corpus and vagina [2-4]. Mesonephric-like carcinoma of the uterine corpus is very rare, with only 32 cases reported to date $[1,5-10]$.

\section{Case Presentation}

A 60-year-old woman with a history of Sjogren's syndrome and menopause at age 50 years was referred to our hospital for further evaluation of a left pelvic mass. Her main symptom was abdominal discomfort lasting for 3 months. She was anemic, with a hemoglobin concentration of $8.3 \mathrm{~g} / \mathrm{ml}$ and a hematocrit of $25.3 \%$. Concentrations of the tumor markers CA125 and CA19-9 were elevated, to $227.8 \mathrm{U} / \mathrm{mL}$ (normal range $<35 \mathrm{U} / \mathrm{ml}$ ) and $83.1 \mathrm{U} / \mathrm{mL}$ (normal range $<33 \mathrm{U} / \mathrm{ml}$ ), respectively. Pelvic magnetic resonance imaging (MRI) showed a $7.0 \times 6.7 \times 6.3 \mathrm{~cm}$ sized, well-enhanced papillary mass in her left ovary accompanied by an internal hemorrhagic component, suggesting a ma- lignant ovarian tumor (Figure 1). Pelvic MRI also showed multiple myoma uteri and ascites. A whole body bone scan showed no evidence of bone metastasis, and the results of colonoscopy, endoscopy and chest computed tomography were unremarkable.

Surgery revealed a protruding tumor on the left side of her uterus, but no sign of a mass in her left ovary. The patient underwent total abdominal hysterectomy, bilateral salpingo-oophorectomy, pelvic and para-aortic lymph node dissection, and partial omentectomy.

Microscopically, most of the tumor was located in the deep myometrium of the uterine corpus and extended to the fundus, left periadnexal soft tissue and broad ligament. The tumor was found to invade the myometrium, but did not involve the endometrium or cervix (Figure 2A). Both adnexa, as well as the peritoneum and omentum, were intact and without metastasis. The tumor was composed of cells with various growth patterns, including tubulocystic, ductal, cribriform, retiform and solid patterns with packed small tubules (Figure 2B-2F). Most of the tumor showed a solid pattern with packed small tubules (Figure 2B). Dense eosinophilic intraluminal secretions were occasionally observed in some of the areas showing tubulocystic and cribriform patterns (Figure 2C).

Immunohistochemistry showed that the tumor cells were diffusely positive for cytokeratin (AE1/AE3), CD10, GATA3, PAX8, PAX2, vimentin, and TTF-1 (Figure 3A$3 \mathrm{~F})$. The apical luminal borders of the glandular structures were positive for $\mathrm{CD} 10$, with the structures being focally
Eur. J. Gynaecol. Oncol. - ISSN: 0392-2936 XLI, n. 5, 2020

doi: $10.31083 /$ j.ejgo.2020.05.5420
This is an open access article under the CC BY 4.0 license (https://creativecommons.org/licenses/by/4.0/). 


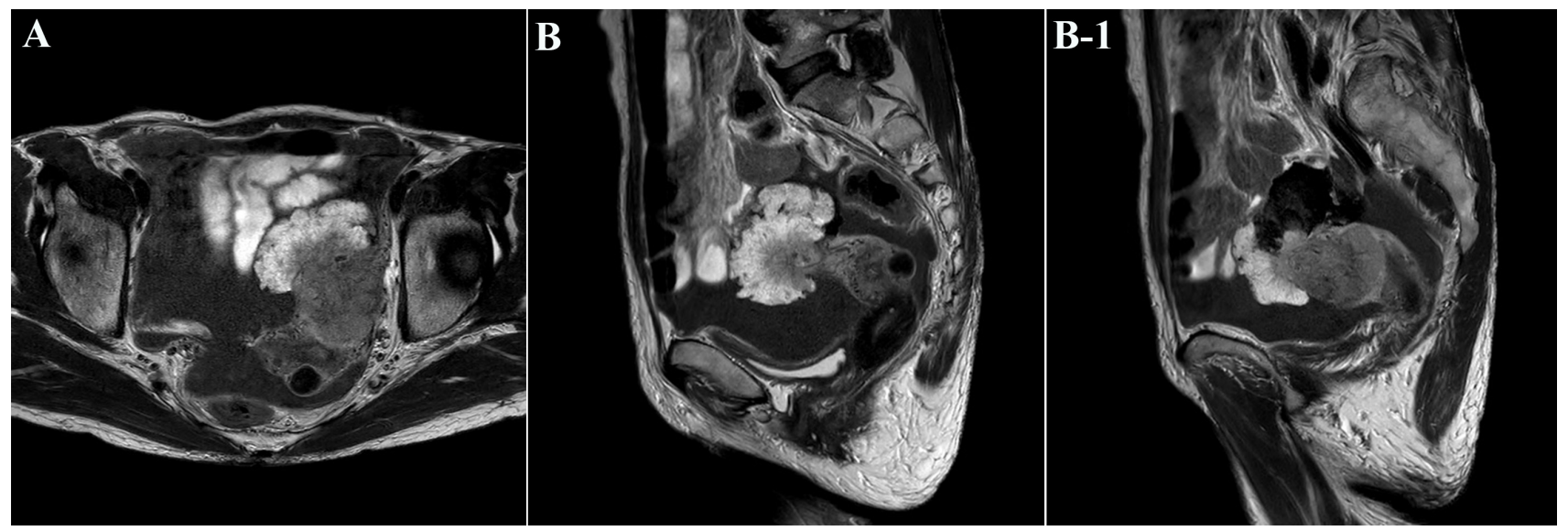

Figure 1. - MRI T2 weighted images showed a $7.0 \times 6.7 \times 6.3 \mathrm{~cm}$ sized papillary growing mass with internal hemorrhagic component arising from the left ovary. The mass was attached to the uterine fundus. Ascites and peritoneal thickening were observed. A. Axial view, B. Sagittal views. M: mass, U: uterus.

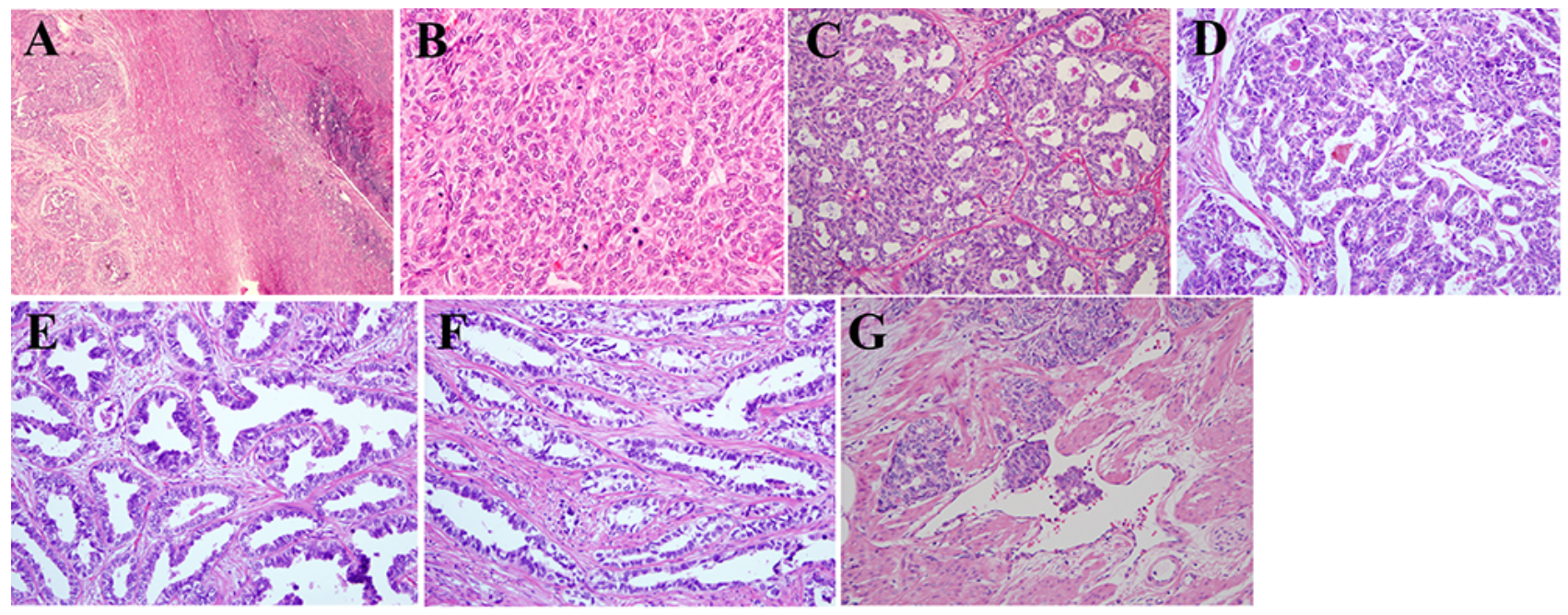

Figure 2. - H\&E staining (magnification). A. Myometrium invasion without involvement of the endometrium $(\times 400)$; B. Predominantly solid pattern with closely packed small tubules, note the increased mitotic activity up to $8 / \mathrm{HPF}(\times 400)$; C. Cribriform, note the dense eosinophilic intraluminal secretions $(\times 200)$; D. Retiform areas mimicking endometrioid carcinoma $(\times 200)$; E. Tubulocystic architecture with focal superficial papillary structures $(200 \times)$; F. Ductal pattern $(\times 200)$; G. Lymphovascular invasion was frequently identified $(\times 400)$.

positive for EMA (Figure 3A, 3G), an immunoreactivity pattern suggesting that the tumor was of mesonephric origin [11]. Tumor cells were negative for ER, WT1, inhibin, CK20, p16, synaptophysin, CD56a and chromogranin, confirming the diagnosis of mesonephric adenocarcinoma. Lymphovascular invasion was frequently observed (Figure 2G). One lymph node was positive for metastasis. The final diagnosis was mesonephric-like carcinoma, stage pT3N1M. After the operation, the serum concentrations of CA125 and CA19-9 decreased to $50.5 \mathrm{U} / \mathrm{mL}$ and $32.6 \mathrm{U} / \mathrm{mL}$, respectively. Following surgery, the patient was treated with adjuvant cisplatin-adriamycin chemotherapy.

\section{Discussion}

Mesonephric-like carcinoma is a rare tumor of the female genital tract, mainly occurring in the cervix and vagina [2]. Diagnosing mesonephric-like carcinoma of the uterine corpus is pathologically challenging because these tumors are rare and present with a wide spectrum of morphologic patterns. Thus, these tumors are frequently confused with other tumor types, including endocervical adenocarcinoma, endometrioid carcinoma, high grade serous carcinoma and sex cord stromal tumor [11]. Positive immunoreactivity for GATA-3, PAX8, PAX2 and CD10 (luminal), and negative immunostaining for estrogen receptors are features supportive of mesonephric-like carcinoma.

In the patient described here, the tumor was located on 


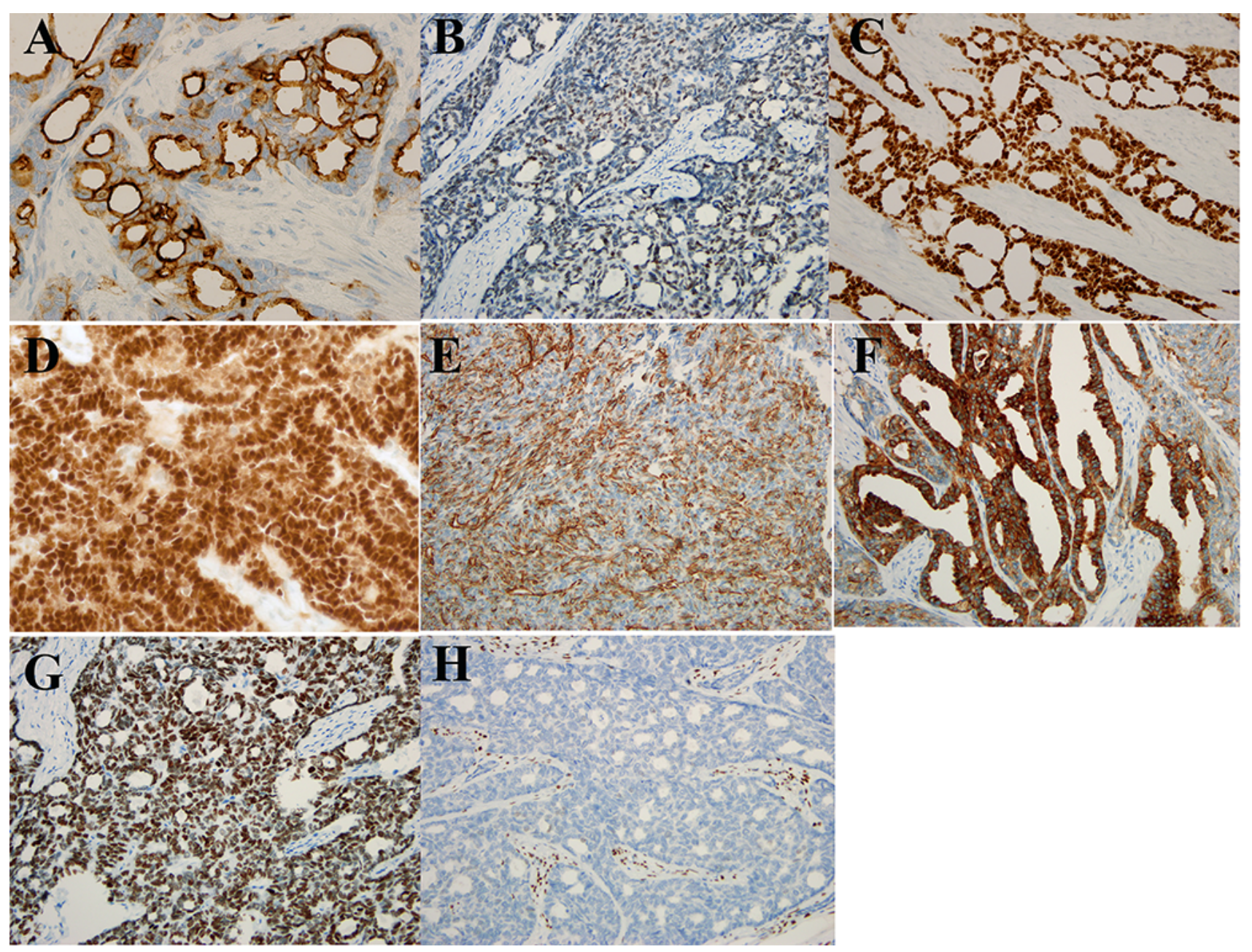

Figure 3. - Immunohistochemical staining. A. Positive immunoreactivity for CD10 along the luminal border $(\times 200)$; B. Weak positive immunoreactivity for GATA3 $(\times 200)$; C. Positive immunoreactivity for PAX8 $(\times 200)$; D. Positive immunoreactivity for PAX2 $(\times 200)$; E. Positive immunoreactivity for vimentin $(\times 200)$; F. Focal positive immunoreactivity for EMA $(\times 200)$; G. Positive immunoreactivity for TTF1 (× 200); H. Negative immunoreactivity for ER $(\times 200)$.

the lateral side of the uterine corpus, but did not involve the endometrium and or adnexa. The broad spectrum of its morphologic features, combined with the presence of GATA3, PAX8, and PAX2 and the absence of hormone receptors on immunohistochemistry, confirmed the diagnosis of mesonephric-like carcinoma.

Differential diagnoses of mesonephric-like carcinoma include female adnexal tumor of probable Wolffian origin (FATWO) and carcinomas of endometrial origin, such as endometrioid, serous, and clear cell carcinomas. Because the tumor in this patient was characterized by cells with a diversity of morphologic patterns and tumor locations in the upper uterine corpus and broad ligament, FATWO was considered the most likely clinical entity. Most FATWOs, however, are immunohistochemically positive for ERs and negative for PAX8, PAX2, and GATA3, thus ruling out the possibility of FATWO in the present case. Negative immunohistochemical staining for inhibin, WT-1 and CD56a excluded sex cord stromal tumor. Tumors of endometrial ori- gin were considered less likely, as the entire endometrium was intact without tumor involvement, and there was no evidence of adenomyosis or endometriosis involving the uterus. Despite the tumor having tubular and glandular features, exhibiting luminal positivity for CD10, endometrioid carcinoma was ruled out due to tumor positivity for PAX8 and GATA3 and negativity for ER (Figure 3H). Endometrial serous carcinoma was considered a possibility due to the papillary and tubulocystic architectural patterns of the tumor, its relatively high grade of nuclear atypia and its immunohistochemical positivity for $\mathrm{p} 53$. However, the tumor epicenter was located in the deep myometrium, which was separate from the endometrium and was immunohistochemically positive for GATA3 and patchy positive for p16, ruling out the possibility of endometrial serous carcinoma. The diversity of architectural pattern, the presence of eosinophilic intraluminal secretion into the cytoplasm and ER negativity suggested the possibility of an endometrial clear cell carcinoma. However, the positivity for GATA3 
and PAX8 and negativity for Napsin-A suggested a cancer other than clear cell carcinoma. The tumor cells were immunohistochemically positive for TTF-1, with the intensity of immunoreactivity being diffuse. In contrast, the tumor showed relatively weak staining for GATA3. Although previous studies of mesonephric carcinoma suggested that TTF-1 and GATA3 had an inverse staining pattern [10, 12], this pattern was not observed in our patient. In addition, mesonephric-like carcinoma should be considered in the differential diagnosis of an unusually presenting tumor along the lateral side of the uterus containing cells with a broad spectrum of histologic patterns. Despite most cells showing a predominant growth pattern, various growth patterns have been observed in these tumors $[5,13]$. Tumor diagnosis can be confirmed by positive immunohistochemical staining of tumor cells for PAX8 and GATA3, a pattern of luminal positivity for CD10 and negativity for ER [5, 14].

Because mesonephric-like carcinomas are very rare, their treatment has not been standardized, with treatment recommendations based on episodic reports. Radical surgery may be curative, particularly when malignant lesions appear well capsulated [15]. Depending on tumor location, radiochemotherapy should be considered if surgery would lead to poor functional outcomes. Response and prognosis are unclear, although adjuvant therapy can be considered [16].

\section{Ethics approval and consent to participate}

All Subjects gave their informed consent for inclusion before they participated in the study. The study was conducted in accordance with Declaration of Helsinki, and the protocol was approved by the Ethics Committee of St. Vincent's Hospital (approval number: VC19ZESI0135).

\section{Acknowledgements}

This work was supported by the National Research Foundation of Korea (NRF) grant funded by the Korea government (NRF 2017R1D1A1B3030021, NRF 2019R1A2C1086807).

The author thanks to Dr. Hyunsoo Kim, Dr. Ahwon Lee and Dr. SoyoungIm for their dedicated advice and assistance in diagnosis this case.

\section{Conflict of Interest}

None of the authors reported financial disclosures.

Submitted: October 29, 2019

Accepted: April 27, 2020

Published: October 15, 2020

\section{References}

[1] Marquette A., Moerman P., Vergote I., Amant F.: "Second case of uterine mesonephric adenocarcinoma". Int. J. Gynecol. Cancer, 2006, 16, 1450-1454.

[2] Ferry J.A., Scully R.E.: "Mesonephric Remnants, Hyperplasia, and Neoplasia in the Uterine Cervix". Am. J. Surg. Pathol., 1990, 14, 1100-1111.

[3] Bagué S., Rodríguez I.M., Prat J.: "Malignant Mesonephric Tumors of the Female Genital Tract". Am. J. Surg. Pathol., 2004, 28, 601607.

[4] Wu H., Zhang L., Cao W., Hu Y., Liu Y.: "Mesonephric adenocarcinoma of the uterine corpus". Int. J. Clin. Exp. Pathol., 2015, 7, 7012-7019.

[5] Zhang L., Cai Z., Ambelil M., Conyers J., Zhu H.: "Mesonephric Adenocarcinoma of the Uterine Corpus". Int. J. Gynecol. Pathol., 2019, 38, 224-229.

[6] Yamamoto Y., Akagi A., Izumi K., Kishi Y.: "Carcinosarcoma of the uterine body of mesonephric origin". Pathol. Int., 1995, 45, 303309.

[7] Ordi J., Nogales F.F., Palacin A., Márquez M., Pahisa J., Vanrell J.A., et al.: "Mesonephric Adenocarcinoma of the Uterine Corpus". Am. J. Surg. Pathol., 2001, 25, 1540-1545.

[8] Ando H., Watanabe Y., Ogawa M., Tamura H., Deguchi T., Ikeda K., et al.: "Mesonephric adenocarcinoma of the uterine corpus with intracystic growth completely confined to the myometrium: a case report and literature review”. Diagn. Pathol., 2017, 12, 63.

[9] Na K., Kim H.: "Clinicopathologic and Molecular Characteristics of Mesonephric Adenocarcinoma Arising From the Uterine Body". Am. J. Surg. Pathol., 2019, 43, 12-25.

[10] Kim S.S., Nam J.H., Kim G., Choi Y.D., Choi C., Park C.S. "Mesonephric Adenocarcinoma of the Uterine Corpus". Int. J. Gynecol. Pathol., 2016, 24, 153-158.

[11] Silver S.A., Devouassoux-Shisheboran M., Mezzetti T.P., Tavassoli F.A.: "Mesonephric Adenocarcinomas of the Uterine Cervix". Am. J. Surg. Pathol., 2001, 25, 379-387.

[12] Pors J., Cheng A., Leo J.M., Kinloch M.A., Gilks B., Hoang L.: “A Comparison of GATA3, TTF1, CD10, and Calretinin in Identifying Mesonephric and Mesonephric-like Carcinomas of the Gynecologic Tract". Am. J. Surg. Pathol., 2018, 42, 1596-1606.

[13] Clement P.B., Young R.H., Keh P., Östör A.G., Scully R.E.: “A Report of Eight Cases, Including Four with a Malignant Spindle Cell Component”. Am. J. Surg. Pathol., 1995, 19, 1158-1171.

[14] Howitt B.E., Emori M.M., Drapkin R., Gaspar C., Barletta J.A., Nucci M.R., et al.: "GATA3 Is a Sensitive and Specific Marker of Benign and Malignant Mesonephric Lesions in the Lower Female Genital Tract”. Am. J. Surg. Pathol., 2015, 39, 1411-1419.

[15] Bifulco G., Mandato V.D., Mignogna C., Giampaolino P., Di Spiezio Sardo A., De Cecio R., et al.: "A case of mesonephric adenocarcinoma of the vagina with a 1-year follow-up". Int. J. Gynecol. Cancer, 2008, 18, 1127-1131.

[16] Mueller I., Kametriser G., Jacobs V.R., Bogner G., Staudach A., Koch H., et al.: "Mesonephric adenocarcinoma of the vagina". Strahlenther. Onkol., 2016, 192, 668-671.

Corresponding Author:

DONG CHOON PARK, M.D., Ph.D.

Department of Obstetrics and Gynecology, Saint Vincent's Hospital, The Catholic University of Korea, 93, Jungbu-daero, Paldal-gu, Suwon-si, Gyeonggi-do (South Korea)

e-mail: park.dongchoon@gmail.com 